\title{
Computing for Eigenpairs on Globally Convergent Iterative Method for Hermitian Matrices
}

\author{
Ran Baik ${ }^{1}$, Karabi Datta ${ }^{2}$, and Yoopyo Hong ${ }^{2}$ \\ 1 Department of Computer Engineering, Honam University, \\ Gwangju 506-090, Korea \\ 2 Department of Mathematical Sciences, Northern Illinois University, \\ DeKalb, IL 60115, USA \\ baik@honam.ac.kr, \{dattak, hong\}@math.niu.edu
}

\begin{abstract}
Let $A=A^{*} \in M_{n}$ and $\mathcal{L}=\left\{\left(U_{k}, \lambda_{k}\right) \mid U_{k} \in \mathbb{C}^{n},\left\|U_{k}\right\|=\right.$ 1 and $\left.\lambda_{k} \in \mathbb{R}\right\}$ for $k=1, \cdots, n$ be the set of eigenpairs of $A$. In this paper we develop a modified Newton method that converges to a point in $\mathcal{L}$ starting from any point in a compact subset $\mathcal{D} \subseteq \mathbb{C}^{n+1}, \mathcal{L} \subseteq \mathcal{D}$.
\end{abstract}

\section{Introduction}

We denote by $M_{n}$ the space of $n$-by- $n$ complex matrices. We denote by $\sigma(A)$ the set of eigenvalues of $A \in M_{n}$. Let $A \in M_{n}$ be Hermitian. Then there is a unitary $U=\left[U_{1} \cdots U_{n}\right] \in M_{n}$ such that $A=U\left[\begin{array}{ccc}\lambda_{1} & & 0 \\ & \ddots & \\ 0 & & \lambda_{n}\end{array}\right] U^{*}, \lambda_{k} \in \mathbb{R}$.

We assume that the eigenvalues $\lambda_{k}$ of $A$ are arranged in decreasing order, i.e., $\lambda_{1} \geq \cdots \geq \lambda_{n}[3$, chapter 4$]$.

Let $\mathcal{L}=\left\{\left[\begin{array}{l}U_{k} \\ \lambda_{k}\end{array}\right] \mid U_{k} \in \mathbb{C}^{n},\left\|U_{k}\right\|=1, \quad \text { and } \lambda_{k} \in \mathbb{R}\right\}_{k=1, \ldots, n} \quad$ be the set of eigenpairs of $A$, and suppose $\mathcal{D}=\left\{\left[\begin{array}{l}X \\ \alpha\end{array}\right] \mid X \in \mathbb{C}^{n},\|X\|_{2}=1\right.$, and $\left.\alpha \in[a, b]\right\}$ be a compact subset of $\mathbb{C}^{n} \times \mathbb{R}$ such that $\mathcal{L} \subseteq \mathcal{D}$. The purpose of this paper is to compute on globally convergent iteration method which converges to an eigenpair of $A$, i.e., an element of $\mathcal{L}$, starting from any arbitrary point $\left[\begin{array}{c}X \\ \alpha\end{array}\right] \in \mathcal{D}$. The following is the usual Newton method for obtaining an eigenpair of a hermitian matrix $A \in M_{n}[4]:$ Consider $G: \mathbb{C}^{n} \times \mathbb{R} \rightarrow \mathbb{C}^{n} \times \mathbb{R}$ such that

$$
G\left(\left[\begin{array}{c}
X \\
\alpha
\end{array}\right]\right)=\left[\begin{array}{c}
(\alpha I-A) X \\
X^{*} X-1
\end{array}\right]
$$


Then $\mathcal{L}$ is the set of solutions for $G\left(\left[\begin{array}{l}X \\ \alpha\end{array}\right]\right)=0$. Assuming the matrix $\left[\begin{array}{cc}\alpha I-A X \\ 2 X^{*} & 0\end{array}\right]$ $\in M_{n+1}$ is invertible, then the usual newton iteration is

$$
\left[\begin{array}{c}
X^{\prime} \\
\alpha^{\prime}
\end{array}\right]=\left[\begin{array}{c}
X \\
\alpha
\end{array}\right]-\left[\begin{array}{cc}
\alpha I-A X \\
2 X^{*} & 0
\end{array}\right]^{-1}\left[\begin{array}{c}
(\alpha I-A) X \\
X^{*} X-1
\end{array}\right]
$$

It is well known that the newton's method has a local quadratic convergence rate [2], that is there is a small neighborhood $N_{\in_{k}}$ for each eigenpair $\left[\begin{array}{c}U_{k} \\ \lambda_{k}\end{array}\right]$ such that if $\left[\begin{array}{c}X \\ \alpha\end{array}\right] \equiv\left[\begin{array}{c}X^{(0)} \\ \alpha^{(0)}\end{array}\right] \in N_{\in_{k}}$ then $\left\|\left[\begin{array}{c}X^{(i+1)} \\ \alpha^{(i+1)}\end{array}\right]-\left[\begin{array}{c}U_{k} \\ \lambda_{k}\end{array}\right]\right\|_{2} \leq C\left\|\left[\begin{array}{c}X^{(i)} \\ \alpha^{(i)}\end{array}\right]-\left[\begin{array}{c}U_{k} \\ \lambda_{k}\end{array}\right]\right\|_{2}^{2}$ for all $i=0,1, \cdots$, where $C<\infty$ is a positive constant.

We call $N_{\in_{k}}$ the quadratic convergence neighborhood of the eigenpair $\left[\begin{array}{c}U_{k} \\ \lambda_{k}\end{array}\right]$. Although the specific determination of each $N_{\in_{k}}$ is an extremely difficult task, if the method converges to a point in $\mathcal{L}$ then we know the rate of convergence will eventually be quadratic. It can be shown easily that the newton's method is not global. We provide an example:

Example 1. Let $A=\left[\begin{array}{cc}1.1+\epsilon & 0 \\ 0 & 0.9\end{array}\right], \epsilon>0$ be the objective matrix with eigenpairs $\left[\begin{array}{l}U_{1} \\ \lambda_{1}\end{array}\right]=\left[\begin{array}{c}1 \\ 0 \\ 1.1+\epsilon\end{array}\right]$ and $\left[\begin{array}{c}U_{2} \\ \lambda_{2}\end{array}\right]=\left[\begin{array}{c}0 \\ 1 \\ 0.9\end{array}\right]$. Suppose the initial points are $\left[\begin{array}{l}X_{1} \\ \alpha_{1}\end{array}\right]=\left[\begin{array}{c}\frac{1}{\sqrt{2}} \\ \frac{1}{\sqrt{2}} \\ 1\end{array}\right]$ and $\left[\begin{array}{l}X_{2} \\ \alpha_{2}\end{array}\right]=\left[\begin{array}{c}\frac{-1}{\sqrt{2}} \\ \frac{1}{\sqrt{2}} \\ 1\end{array}\right]$.

Then the newton iteration (2) becomes

$$
\begin{aligned}
& {\left[\begin{array}{c}
X_{1}^{\prime} \\
\alpha_{1}^{\prime}
\end{array}\right]=\left[\begin{array}{c}
X_{1} \\
\alpha_{1}
\end{array}\right]-\left[\begin{array}{cc}
\alpha_{1} I-A X_{1} \\
2 X_{1}^{*} & 0
\end{array}\right]^{-1}\left[\begin{array}{c}
\left(\alpha_{1} I-A\right) X_{1} \\
X_{1}^{*} X_{1}-1
\end{array}\right]} \\
& =\left[\begin{array}{c}
\frac{1}{\sqrt{2}} \\
\frac{1}{\sqrt{2}} \\
1
\end{array}\right]-\left[\begin{array}{ccc}
-0.1-\epsilon & 0 & \frac{1}{\sqrt{2}} \\
0 & 0.1 & \frac{1}{\sqrt{2}} \\
\frac{2}{\sqrt{2}} & \frac{2}{\sqrt{2}} & 0
\end{array}\right]^{-1}\left[\begin{array}{c}
\frac{-(0.1+\epsilon)}{\sqrt{2}} \\
\frac{0.1}{\sqrt{2}} \\
0
\end{array}\right] \\
& =\left[\begin{array}{c}
\frac{1}{\sqrt{2}} \\
\frac{1}{\sqrt{2}} \\
1
\end{array}\right]-1 / \epsilon\left[\begin{array}{ccc}
-1 & 1 & \frac{-0.1}{\sqrt{2}} \\
1 & -1 & \frac{0.1+\epsilon}{\sqrt{2}} \\
\frac{-0.2}{\sqrt{2}} & \frac{2(0.1+\epsilon)}{\sqrt{2}} & -0.1(0.1+\epsilon)
\end{array}\right]\left[\begin{array}{c}
\frac{-(0.1+\epsilon)}{\sqrt{2}} \\
\frac{0.1}{\sqrt{2}} \\
0
\end{array}\right] \\
& =\left[\begin{array}{c}
\frac{1}{\sqrt{2}} \\
\frac{1}{\sqrt{2}} \\
1
\end{array}\right]-1 / \epsilon\left[\begin{array}{c}
\frac{(0.2+\epsilon)}{\sqrt{2}} \\
\frac{-(0.2+\epsilon)}{\sqrt{2}} \\
0.02+0.2 \epsilon
\end{array}\right]
\end{aligned}
$$


Thus if $\epsilon$ goes to zero, the iteration diverges. Similarly, for the initial eigenpair $\left[\begin{array}{l}X_{2} \\ \alpha_{2}\end{array}\right]$. We modify the newton method in order to have a global convergence. There are several considerations to give for the modification. First, under the modification we desire the pair $\left[\begin{array}{c}X^{(i)} \\ \alpha^{(i)}\end{array}\right]$ gets closer to an eigenpair at each step of the iteration, i.e., $d_{\mathcal{L}}\left(\left[\begin{array}{c}X^{(i+1)} \\ \alpha^{(i+1)}\end{array}\right]\right) \leq d_{\mathcal{L}}\left(\left[\begin{array}{c}X^{(i)} \\ \alpha^{(i)}\end{array}\right]\right)$ where $d_{\mathcal{L}}$ is a suitable distance measure from a point to $\mathcal{L}$. It will ensure the points under the iteration remain in $\mathcal{D}$. Second, we want to modify the method the least amount as possible in order to preserve the original properties of the newton's Method, for example, local quadratic convergence. Third, the modified method should be simple to implement, requires almost the same procedures as the original newton iteration.

\section{Modification for Global Newton Iteration}

Consider the newton iteration (2): $\left[\begin{array}{l}X^{\prime} \\ \alpha^{\prime}\end{array}\right]=\left[\begin{array}{c}X \\ \alpha\end{array}\right]-\left[\begin{array}{cc}\alpha I-A X \\ 2 X^{*} & 0\end{array}\right]^{-1}\left[\begin{array}{c}(\alpha I-A) X \\ X^{*} X-1\end{array}\right]$ Then, assuming $\alpha \neq 0$ and $\alpha \notin \sigma(A)$

$$
\begin{aligned}
& {\left[\begin{array}{l}
X^{\prime} \\
\alpha^{\prime}
\end{array}\right]=\left(I-\left[\begin{array}{cc}
\alpha I-A & X \\
2 X^{*} & 0
\end{array}\right]^{-1}\left[\begin{array}{cc}
\alpha I-A & 0 \\
X^{*} & -1 / \alpha
\end{array}\right]\right)\left[\begin{array}{c}
X \\
\alpha
\end{array}\right]} \\
& =\left[\begin{array}{cc}
\alpha I-A & X \\
2 X^{*} & 0
\end{array}\right]^{-1}\left[\begin{array}{cc}
0 & X \\
X^{*} & 1 / \alpha
\end{array}\right]\left[\begin{array}{c}
X \\
\alpha
\end{array}\right] \text {, or }\left[\begin{array}{cc}
\alpha I-A X \\
2 X^{*} & 0
\end{array}\right]\left[\begin{array}{c}
X^{\prime} \\
\alpha^{\prime}
\end{array}\right]=\left[\begin{array}{cc}
0 & X \\
X^{*} & 1 / \alpha
\end{array}\right]\left[\begin{array}{c}
X \\
\alpha
\end{array}\right] \text {. }
\end{aligned}
$$

Choose a parameter $t>0$ so that the method takes the form [1]

$$
\left[\begin{array}{cr}
\alpha I-A & X \\
2 X^{*} & 0
\end{array}\right]\left[\begin{array}{l}
X^{\prime} \\
\alpha^{\prime}
\end{array}\right]=\left[\begin{array}{ll}
I & 0 \\
0 & t
\end{array}\right]\left[\begin{array}{cc}
0 & X \\
X^{*} & 1 / \alpha
\end{array}\right]\left[\begin{array}{c}
X \\
\alpha
\end{array}\right]
$$

Then

$$
(\alpha I-A) X^{\prime}+\alpha^{\prime} X=\alpha X
$$

and

$$
2 X^{*} X^{\prime}=t\left(X^{*} X+1\right)
$$

From (4), we have $X^{\prime}=\left(\alpha-\alpha^{\prime}\right)(\alpha I-A)^{-1} X$, and hence from (5) $X^{*} X^{\prime}=$ $\frac{1}{2} t\left(X^{*} X+1\right)=\left(\alpha-\alpha^{\prime}\right) X^{*}(\alpha I-A)^{-1} X$. Set $\beta \equiv X^{*}(\alpha I-A)^{-1} X$. Then $\frac{t}{2}\left(X^{*} X+1\right)=\beta\left(\alpha-\alpha^{\prime}\right)$, or $\left(\alpha-\alpha^{\prime}\right)=\frac{t}{2}\left(X^{*} X+1\right) \frac{1}{\beta}$. Thus, $X^{\prime}=\frac{t}{2}\left(X^{*} X+\right.$ 1) $\frac{1}{\beta}(\alpha I-A)^{-1} X$, and $\alpha^{\prime}=\alpha-\frac{t}{2}\left(X^{*} X+1\right) \frac{1}{\beta}$. If we normalize the vector $X \in \mathbb{C}^{n}$ in each step of the iteration, $\frac{1}{2}\left(X^{*} X+1\right)=1$.

Thus we have $X^{\prime}=\frac{1}{\left\|\frac{t}{\beta}(\alpha I-A)^{-1} X\right\|_{2}} \frac{t}{\beta}(\alpha I-A)^{-1} X$, and $\alpha^{\prime}=\alpha-\frac{t}{\beta}$. Set $\hat{\beta} \equiv\left\|(\alpha I-A)^{-1} X\right\|_{2}=\left(X^{*}(\alpha I-A)^{-2} X\right)^{\frac{1}{2}}$. 
Then we have $X^{\prime}=\frac{1}{\hat{\beta}} \frac{|\beta|}{\beta}(\alpha I-A)^{-1} X$, and $\alpha^{\prime}=\alpha-\frac{t}{\beta}$. Since $\frac{|\beta|}{\beta}= \pm 1$, we ignore the sign. Then the parameterized newton method takes a form:

$$
\begin{aligned}
X^{\prime} & =\frac{1}{\hat{\beta}}(\alpha I-A)^{-1} X, \text { and } \\
\alpha^{\prime} & =\alpha-\frac{t}{\beta} .
\end{aligned}
$$

Now, suppose $\left[\begin{array}{c}X \\ \alpha\end{array}\right] \in \mathcal{D}$ and Let $\mathcal{L}=\left\{\left[\begin{array}{c}U_{k} \\ \lambda_{k}\end{array}\right] \mid U_{k} \in \mathbb{C}^{n},\left\|U_{k}\right\|_{2}=1\right.$, and $\left.\lambda_{k} \in \mathbb{R}\right\}$ be the set of all eigenpairs of $A$. Define a distance measure from a point $\left[\begin{array}{c}X \\ \alpha\end{array}\right]$ to $\mathcal{L}$ by

$$
d_{\mathcal{L}}\left(\left[\begin{array}{c}
X \\
\alpha
\end{array}\right]\right) \equiv\|(\alpha I-A) X\|_{2}
$$

Clearly, $d_{\mathcal{L}}\left(\left[\begin{array}{c}X \\ \alpha\end{array}\right]\right) \geq 0, d_{\mathcal{L}}\left(\left[\begin{array}{c}X \\ \alpha\end{array}\right]\right)=0$ implies $\left[\begin{array}{c}X \\ \alpha\end{array}\right] \in \mathcal{L}$, and $d_{\mathcal{L}}: \mathcal{D} \rightarrow \mathbb{R}^{+}$ is continuous (since $\mathcal{D}$ is compact, $d_{\mathcal{L}}$ is actually uniformly continuous) [5].

We have the following.

Lemma 1. Let $A \in M_{n}$ be Hermitian. Consider the parameterized newton's method $X^{\prime}=\frac{1}{\hat{\beta}}(\alpha I-A)^{-1} X$ and $\alpha^{\prime}=\alpha-\frac{t}{\beta}$, where $\beta=X^{*}(\alpha I-A)^{-1} X$ and $\hat{\beta}=$ $\left\|(\alpha I-A)^{-1} X\right\|_{2}=\left(X^{*}(\alpha I-A)^{-2} X\right)^{1 / 2}$. Then $d_{\mathcal{L}}\left(\left[\begin{array}{c}X^{\prime} \\ \alpha^{\prime}\end{array}\right]\right)$ is minimized at $t=\left(\frac{\beta}{\hat{\beta}}\right)^{2}$ with $\min _{t} d_{\mathcal{L}}\left(\left[\begin{array}{c}X^{\prime} \\ \alpha^{\prime}\end{array}\right]\right)=\frac{1}{\hat{\beta}}\left(1-\left(\frac{\beta}{\hat{\beta}}\right)^{2}\right)^{\frac{1}{2}}$.

Proof: Suppose $X^{\prime}=\frac{1}{\hat{\beta}}(\alpha I-A)^{-1} X$ and $\alpha^{\prime}=\alpha-\frac{t}{\beta}$. Then

$$
\begin{aligned}
d_{\mathcal{L}}^{2}\left(\left[\begin{array}{l}
X^{\prime} \\
\alpha^{\prime}
\end{array}\right]\right)= & \left\|\left(\left(\alpha-\frac{t}{\beta}\right) I-A\right) \frac{1}{\hat{\beta}}(\alpha I-A)^{-1} X\right\|_{2}^{2} \\
= & \frac{1}{{ }^{2}} X^{*}\left(I-2 \frac{t}{\beta}(\alpha I-A)^{-1}+\left(\frac{t}{\beta}\right)^{2}(\alpha I-A)^{-2}\right) X \\
= & \frac{1}{\hat{\wedge}^{2}}\left(1-2 \frac{t}{\beta} \cdot \beta+t^{2}\left(\frac{\hat{\beta}}{\beta}\right)^{2} .\right.
\end{aligned}
$$

Thus, $d_{\mathcal{L}}^{2}\left(\left(\begin{array}{c}X^{\prime} \\ \alpha^{\prime}\end{array}\right)\right)$ is minimized at $t=\left(\begin{array}{c}\frac{\beta}{\hat{\beta}} \\ { }^{2}\end{array}{ }^{2}\right.$ with $\min _{t} d_{\mathcal{L}}\left(\left[\begin{array}{c}X^{\prime} \\ \alpha^{\prime}\end{array}\right]\right)=$ $\frac{1}{\hat{\beta}}\left(1-\left(\frac{\beta}{\hat{\beta}}\right)^{2}\right)^{\frac{1}{2}}$. 
Therefore, we have the following modification of the newton's method:

$$
\begin{aligned}
X^{\prime} & =\frac{1}{\hat{\beta}}(\alpha I-A)^{-1} X . \\
\alpha^{\prime} & =\alpha-\frac{\beta}{\hat{\beta}^{2}} .
\end{aligned}
$$

The following result shows that the modified iteration (9), (10) is bounded.

Lemma 2. Let $A \in M_{n}$ be a Hermitian such that $\sigma(A)=\left\{\lambda_{1} \geq \cdots \geq \lambda_{n}, \lambda_{k} \in\right.$ $\mathbb{R}\}$. Let $\mathcal{D}=\left\{\left[\begin{array}{l}X \\ \alpha\end{array}\right] \mid X \in \mathbb{C}^{n},\|X\|_{2}=1\right.$, and $\left.\alpha \in[a, b]\right\}$ be such that $a \leq \lambda_{n}$ and $b \geq \lambda_{1}$. Suppose $\mathcal{D}^{\prime}=\left\{\left[\begin{array}{l}X^{(i)} \\ \alpha^{(i)}\end{array}\right]\right\}_{\rangle=1, \infty, \cdots}$ is the sequence of iterates of $(9)$ and (10), i.e., $X^{(i+1)}=\frac{1}{\hat{\beta}^{(i)}}\left(\alpha^{(i)} I-A\right)^{-1} X^{(i)}$, and $\alpha^{(i+1)}=\alpha^{(i)}-\frac{\beta^{(i)}}{\left(\hat{\beta}^{(i)}\right)^{2}}$. Then $\mathcal{D}^{\prime} \subset \mathcal{D}$ whenever $\left[\begin{array}{l}X^{(0)} \\ \alpha^{(0)}\end{array}\right] \in \mathcal{D}$

Theorem 3. Suppose $\mathcal{D}^{\prime}=\left\{\left[\begin{array}{c}X^{(i)} \\ \alpha^{(i)}\end{array}\right]\right\}_{\rangle=1, \infty, \ldots}$ is the sequence of iterates of $(9)$ and (10). Then the sequence $\left\{d_{\mathcal{L}}\left(\left[\begin{array}{l}X^{(i)} \\ \alpha^{(i)}\end{array}\right]\right)\right\}_{i=0,1, \cdots}$ is convergent.

Note from Theorem 3 that since $\left\{d_{\mathcal{L}}\left(\left[\begin{array}{c}X^{(i)} \\ \alpha^{(i)}\end{array}\right]\right)\right\}$ is a monotone decreasing sequence that is bounded below by zero, The sequence $\left\{d_{\mathcal{L}}\left(\left[\begin{array}{c}X^{(i)} \\ \alpha^{(i)}\end{array}\right]\right)\right\}$ converges to either (i) zero, or (ii) a positive constant $L$.

Suppose $\lim _{i \rightarrow \infty} d_{\mathcal{L}}\left(\left[\begin{array}{l}X^{(i)} \\ \alpha^{(i)}\end{array}\right]\right)=0$. Then clearly, $\left\{d_{\mathcal{L}}\left(\left[\begin{array}{c}X^{(i)} \\ \alpha^{(i)}\end{array}\right]\right)\right\}$ converges to an eigenpair of $A$. In the following section we discuss the case $\lim _{i \rightarrow \infty} d_{\mathcal{L}}\left(\left[\begin{array}{c}X^{(i)} \\ \alpha^{(i)}\end{array}\right]\right)=$ $L>0$ which requires some detailed analysis. We summarize the results. Under the modified newton iteration, the sequence $\left\{d_{\mathcal{L}}\right\}$ converges to either zero or $L>0$. If $\left\{d_{\mathcal{L}}\right\}$ converges to $L>0$, then iterates $\left\{\left[\begin{array}{c}X^{(i)} \\ \alpha^{(i)}\end{array}\right]\right\}$ has an accumulation point $\left[\begin{array}{l}X \\ \alpha\end{array}\right]$ where $d_{\mathcal{L}}\left(\left[\begin{array}{l}X \\ \alpha\end{array}\right]\right)=L>0$ such that the point $\alpha \in \mathbb{R}$ lies exactly at the midpoint of two distinct eigenvalues (each eigenvalue may have the algebraic multiplicity more than one) such that corresponding components of the vector $U^{*} X$ have equal weights that are $\frac{1}{2}$ each, see Figure 1 . 


$\begin{array}{ccc}\lambda_{t} & \alpha & \lambda_{s} \\ \left(U^{*} X\right)_{t}^{2}=\frac{1}{2} & & \left(U^{*} X\right)_{s}^{2}=\frac{1}{2}\end{array}$

Fig. 1 .

Therefore, $d_{\mathcal{L}}^{2}=\frac{1}{\hat{\beta}}^{2}=\sum_{k=1}^{n}\left(\alpha-\lambda_{k}\right)^{2}\left|y_{k}\right|^{2}=\left(\alpha-\lambda_{k}\right)^{2}=\frac{\left(\lambda_{s}-\lambda_{t}\right)^{2}}{2}, \quad \lambda_{s}>\lambda_{t}$. We conclude this section with the following consequence of above results and an example.

Theorem 4. Suppose $d_{\mathcal{L}}\left(\left[\begin{array}{l}X \\ \alpha\end{array}\right]\right)=L$. Then both $\alpha+\frac{1}{\hat{\beta}}$ and $\alpha-\frac{1}{\hat{\beta}}$ are the eigenvalues of $A$ and $\frac{1}{\wedge^{\prime}}\left[\left(\alpha+\frac{1}{\wedge}\right) I-A\right]^{-1} X$ and $\frac{1}{\wedge^{\prime \prime}}\left[\left(\alpha-\frac{1}{\wedge}\right) I-A\right]^{-1} X$ are the corresponding eigenvector of $A$, where $\stackrel{\wedge}{\beta}^{\prime}=\left\|\left[\left(\alpha+\frac{1}{\hat{\beta}}\right) I-A\right]^{\beta} X\right\|_{2}^{\beta}$, and $\stackrel{\wedge}{\beta}^{\prime \prime}=\left\|\left[\left(\alpha-\frac{1}{\hat{\beta}}\right) I-A\right]-1 X\right\|_{2}$

Example 2. Consider the Example 1. Let $A=\left[\begin{array}{cc}1.1+\epsilon & 0 \\ 0 & 0.9\end{array}\right], \epsilon>0$. Suppose we start with initial eigenpair $\left[\begin{array}{c}X_{1}^{(0)} \\ \alpha_{1}^{(0)}\end{array}\right]=\left[\begin{array}{c}\frac{1}{\sqrt{2}} \\ \frac{1}{\sqrt{2}} \\ 1\end{array}\right]$. Then

$$
\begin{aligned}
\beta=X^{*}(\alpha I-A)^{-1} X & =\left[\frac{1}{\sqrt{2}} \frac{1}{\sqrt{2}}\right]\left[\begin{array}{cc}
-(0.1-\epsilon) & 0 \\
0 & 0.1
\end{array}\right]^{-1}\left[\begin{array}{c}
\frac{1}{\sqrt{2}} \\
\frac{1}{\sqrt{2}}
\end{array}\right] \\
& =\left[\frac{1}{\sqrt{2}} \frac{1}{\sqrt{2}}\right]\left[\begin{array}{cc}
-1 /(0.1+\epsilon) & 0 \\
0 & 10
\end{array}\right]\left[\begin{array}{c}
\frac{1}{\sqrt{2}} \\
\frac{1}{\sqrt{2}}
\end{array}\right] \\
& =\frac{-1}{2(0.1+\epsilon)}+5=5-\frac{1}{0.2+2 \epsilon}, \text { and }
\end{aligned}
$$

$$
\begin{aligned}
\hat{\beta}=\left\|(\alpha I-A)^{-1} X\right\|_{2} & =\left\|\left[\begin{array}{cc}
-1 /(0.1+\epsilon) & 0 \\
0 & 10
\end{array}\right]\left[\begin{array}{c}
\frac{1}{\sqrt{2}} \\
\frac{1}{\sqrt{2}}
\end{array}\right]\right\|_{2} \\
& =\left(\frac{1}{2}\left(\frac{-1}{0.1+\epsilon}\right)^{2}+50\right)^{1 / 2}=\left(\frac{1}{0.02+0.4 \epsilon+\epsilon^{2}}+50\right)^{1 / 2} .
\end{aligned}
$$


If $\epsilon$ goes to zero, then $\beta \rightarrow 0$ and $\hat{\beta} \rightarrow 10$. Notice that for $\epsilon \cong 0$

$$
d_{\mathcal{L}}\left(\left[\begin{array}{c}
X^{(0)} \\
\alpha^{(0)}
\end{array}\right]\right)=d_{\mathcal{L}}\left(\left[\begin{array}{c}
X^{(m)} \\
\alpha^{(m)}
\end{array}\right]\right)=\ldots=0.1
$$

Therefore by Theorem 4 , we have $d_{\mathcal{L}}\left(\left[\begin{array}{c}X^{(n)} \\ \alpha^{(n)}\end{array}\right]\right)=\frac{1}{\hat{\beta}}$.

Hence $\lambda_{1}(A)=1+1 / \hat{\beta}=1+.1=1.1$ and $\lambda_{2}(A)=1-1 / \hat{\beta}=1-.1=.9$.

We obtain $X_{1}=\left[\begin{array}{l}1 \\ 0\end{array}\right]$ and $X_{2}=\left[\begin{array}{l}0 \\ 1\end{array}\right]$ by solving $\left(A-\lambda_{1} I\right) X_{1}=0$ and $(A-$ $\left.\lambda_{2} I\right) X_{2}=0$.

\section{Examples with Modified Newton's Iterations}

Example 3. Let $H=\left[\begin{array}{ccccc}1 & \frac{1}{2} & \frac{1}{3} & \cdots & \frac{1}{n} \\ \frac{1}{2} & \frac{1}{3} & & & \vdots \\ \vdots & & \ddots & & \\ \vdots & & & \ddots & \\ \frac{1}{n} & \frac{1}{n+1} & \cdots & \cdots & \frac{1}{2 n-1}\end{array}\right]$ be a $n$ by $n$ Hilbert matrix $H$.

The Hilbert matrix is a well-known example of an ill-conditioned positive definite matrix. Because the smallest eigenvalue $\lambda_{12}$ of $H \in M_{12}$ is so near zero, many conventional algorithms produce $\lambda_{12}=0$. Our method gives the following expermental results: Set the convergence criterion, $\epsilon=2 \times 10^{-16}$, i.e., $\left\|\left(H-\alpha_{k}^{(i)} I\right) X_{k}^{(i)}\right\|_{2}<\epsilon$.

Suppose $\mathcal{D}=\left\{\left[\begin{array}{c}X \\ \alpha\end{array}\right] \mid X \in\left\{e_{1}, \cdots, e_{12}\right\}, \quad\right.$ and $\left.\quad \alpha \in\left\{h_{1,1}, \cdots, h_{12,12}\right\}\right\}$ is the initial set of points where $e_{i}$ is the $i$ th column of the identity matrix and $h_{i, i}$ is the $i$ th diagonal entry of $H$.

Table 1. Eigenvalues of $H_{12}$ by Modified Newton Iteration

\begin{tabular}{|c|c|c|}
\hline Eigenvalues & Eigenvalues of $H$ & $\left\|\left(H-\alpha_{k}^{(i)} I\right) X_{k}^{(i)}\right\|_{2}$ \\
\hline 1st & 1.7953720595620 & $4.5163365159057 \mathrm{D}-17$ \\
\hline 2nd & 0.38027524595504 & $9.5107769421299 \mathrm{D}-17$ \\
\hline 3rd & $4.4738548752181 \mathrm{D}-02$ & $9.3288777118150 \mathrm{D}-17$ \\
\hline 4th & $3.7223122378912 \mathrm{D}-03$ & $9.5107769421299 \mathrm{D}-17$ \\
\hline th & $2.3308908902177 \mathrm{D}-04$ & $6.5092594645258 \mathrm{D}-17$ \\
\hline 6th & $1.1163357483237 \mathrm{D}-05$ & $6.6374428417771 \mathrm{D}-17$ \\
\hline 7th & $4.0823761104312 \mathrm{D}-07$ & $1.9236667674542 \mathrm{D}-16$ \\
\hline 8th & $1.1228610666749 \mathrm{D}-08$ & $4.9553614188006 \mathrm{D}-17$ \\
\hline 9th & $2.2519644461451 \mathrm{D}-10$ & $6.0015952254039 \mathrm{D}-17$ \\
\hline 10 th & $3.1113405079204 \mathrm{D}-12$ & $6.5125904614112 \mathrm{D}-17$ \\
\hline 11 th & $2.6487505785549 \mathrm{D}-14$ & $1.0932505712948 \mathrm{D}-16$ \\
\hline 12 th & $1.1161909467844 \mathrm{D}-16$ & $6.0015952254039 \mathrm{D}-17$ \\
\hline
\end{tabular}


Example 4. Let $H_{3}=\left[\begin{array}{ccc}10^{40} & 10^{19} & 10^{19} \\ 10^{19} & 10^{20} & 10^{9} \\ 10^{19} & 10^{9} & 1\end{array}\right]$ be a 3 by 3 graded matrix. Suppose $\mathcal{D}=\left\{\left[\begin{array}{c}X \\ \alpha\end{array}\right] \mid X \in\left\{e_{1}, e_{2}, e_{3}\right\}, \quad\right.$ and $\left.\quad \alpha \in\left\{h_{1,1}, h_{2,2}, h_{3,3}\right\}\right\}$ is the initial set of points where $e_{i}$ is the $i$ th column of the identity matrix and $h_{i, i}$ is the $i$ th diagonal entry of $H$. The graded matrix is a well-known example of an ill-conditioned symmetric matrix. The following is the result obtained by MATLAB and our method.

Table 2. Eigenvalues of $H_{3}$ by Modified Newton Iteration

\begin{tabular}{|c|c|c|c|}
\hline & \multicolumn{2}{|c|}{ Modified Newton Method } & MATLAB \\
\hline Eigenvalues & Eigenvalues of $H$ & Iteration & Eigenvalues of $H$ \\
\hline$\lambda_{1}$ & $1.0000000000000 \mathrm{D}+40$ & 2 & $9.999999999999999 \mathrm{e}+39$ \\
\hline$\lambda_{2}$ & $1.0000000000000 \mathrm{D}+20$ & 1 & 0 \\
\hline$\lambda_{3}$ & 0.98000000000020 & 2 & $-1.000000936789517+20$ \\
\hline
\end{tabular}

\section{References}

1. Karabi Datta and Yoopyo Hong and Ran Baik Lee : Parameterized Newton's iteration for computing an Eigenpairs of a Real Symmetric Matrix in an Interval, Computational Methods in Applied Mathemaics, 3.(2003) 517-535

2. R. A. Horn and C. R. Johnson,: Matrix Analysis, Cambridge University Press, (1985).

3. J. M. Ortega: Numerical Analysis, A Second Course. SIAM Series in Classical in Applied Mathematics, Philadelphia, SIAM Publications, (1990).

4. J. M. Ortega and W. C. Rheinboldt: Iterative Solution Of Nonlinear Equations In Several Variables, Academic Press, New York and London, (1970).

5. H.L. Royden: Real Analysis, Macmillan Publishing Company, New York,(1968). 